\title{
Skin Impedance Models for Transdermal Drug Delivery
}

\author{
Grantham K.H. Pang and DaPeng Qiao \\ Department of Electrical and Electronic Engineering, \\ Industrial Automation Research Laboratory, \\ The University of Hong Kong, Pokfulam Road, Hong Kong \\ gpang@eee.hku.hk
}

\begin{abstract}
In this paper, a study based on the different models of the skin impedance is carried out. The purpose is to examine the drug delivery method through iontophoresis, which relies on active transportation of the charged medication agent within an electric field. It is a kind of transdermal drug delivery method, and hence the method has to handle the variability in skin characteristics of a patient. This paper carries out a simulation study based on three different skin impedance models.
\end{abstract}

Index Terms - Target drug delivery, biomedical engineering, skin impedance, iontophoresis.

\section{INTRODUCTION}

Nowadays, as opposed to the traditional oral intake and hypodermal injection, drug delivery to a patient can be carried out in many different ways. Transdermal drug delivery releases the medicament into the patient's body via the skin. There are various methods for transdermal drug delivery and they have been developed based on various principles. The developed methods could be based on diffusion, absorption, thermal energy, radio frequency energy, ultrasound, electrostatic force (electrophoresis) or electric field (iontophoresis). These are non-invasive methods, and recent developments also include transdermal skin patch that is placed on the skin to deliver a specific dose of medication through the skin, and then into the blood stream of the patient. The main advantage of a transdermal drug delivery is that it provides a controlled release of the medicament without serious pain to the patient.

However, all transdermal drug delivery methods have to deal with the complicated properties of the patient' skin, which is a very effective barrier, and its characteristics can vary a lot from one person to another. It is a natural barrier to foreign chemicals and biological agents. Methods that are based on the use of microneedles [1,2,3] have also been developed. These microneedles would physically puncture the skin but for less than $1 \mathrm{~mm}$ and deliver the drug without piercing blood vessels or damaging nerves, that are typically around $1 \mathrm{~mm}$ under the skin surface. The mechanical

Grantham .K.H. Pang is with the Department of Electrical and Electronic Engineering, The University of Hong Kong (e-mail: gpang@eee.hku.hk). stability and the puncture behaviour of microneedles have been investigated experimentally [3]. In this paper, several electrical models for skin impedance are investigated and simulations have been carried out for a comparative study of the parameters for drug delivery through iontophoresis [4, $5]$.

\section{METHOD AND ANALYSIS}

Research has found that skin impedance can be modeled by typical RC (resistor-capacitor) circuits, without the need of any inductive component $[6,7,8,9]$. Hence, it is generally agreed that the skin impedance is made up of some amount of resistance and some form of capacitance. Different models of RC circuits can be developed to simulate current responses in actual skin.

\section{$\underline{\text { Model A }}$}

An early skin impedance model is given in [7] and it is shown in Figure 1. The model is made up of a capacitor $\mathrm{C}$ in parallel with the resistance $R_{1}, R_{1}$ is modeling the resistance of the stratum corneum, which is the top layer of the epidermis. $\mathrm{C}$ represents the capacitance of the skin. The $C-R_{1}$ parallel combination is in series with another resistance $\mathrm{R}_{2}$ which represents the resistance of the deeper tissues within the epidermis. Example of value for $R_{1}$ would range from around $100 \Omega$ to $5000 \mathrm{k} \Omega \mathrm{cm}^{2}$, while $\mathrm{R}_{2}$ would range from around $0.1 \Omega$ to $1.0 \mathrm{k} \Omega \mathrm{cm}^{2}$. This model is simple and can serve as an initial representation to the skin characteristics. However, a more complete representation is given in the next subsection.

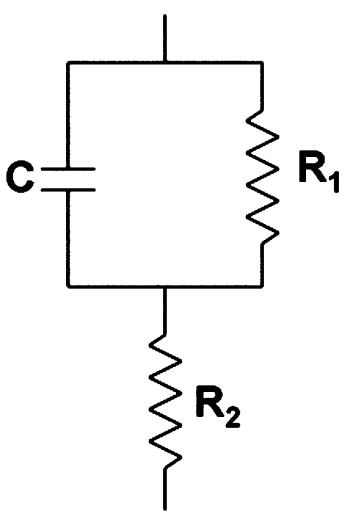

Fig. 1 Skin Impedance Model A 
Model B

Another model can also be found in [8] by Tregear which is consisted of multiple parallel RC circuits in series. This is designed to model the varying capacitance and resistance of the epidermal skin layers at different depths of the skin. This model is shown in Figure 2 below. As mentioned in [5], this model represents the decreasing values of capacitance and resistance as individual layers of the stratum corneum are removed in experiments. The experimental result was important as it allows for the development of a more precise model of the skin impedance. Also, it has shown that the stratum corneum accounts for the major portion of the skin impedance in the skin.

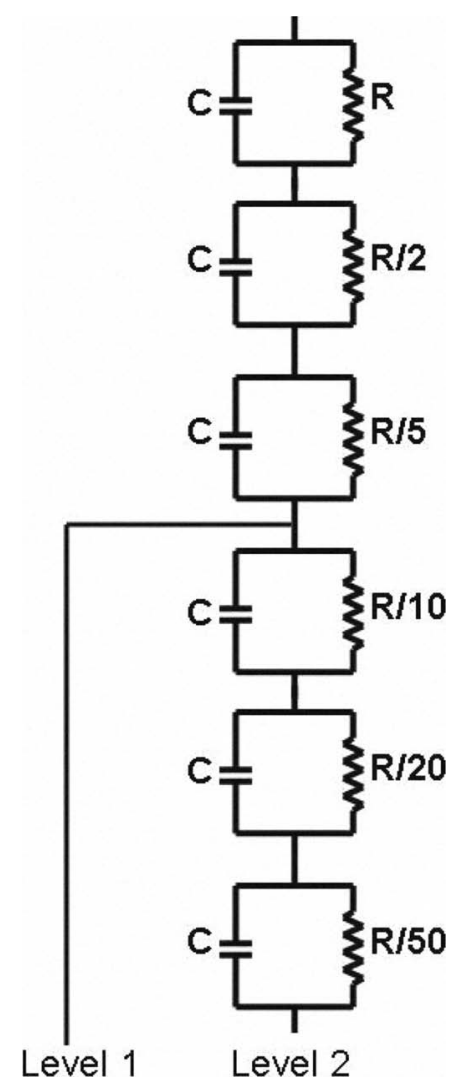

Fig. 2 Skin Impedance Model B

\section{Model C}

Lykken [9] has proposed another model of skin impedance as shown in Figure 3. The model consists of several parallel paths, and each path is made up of several RC circuits, with each circuit representing a different layer of the skin. Figure 3 provides a more distributed nature when modeling the skin impedance, and can potentially provide a more accurate model.

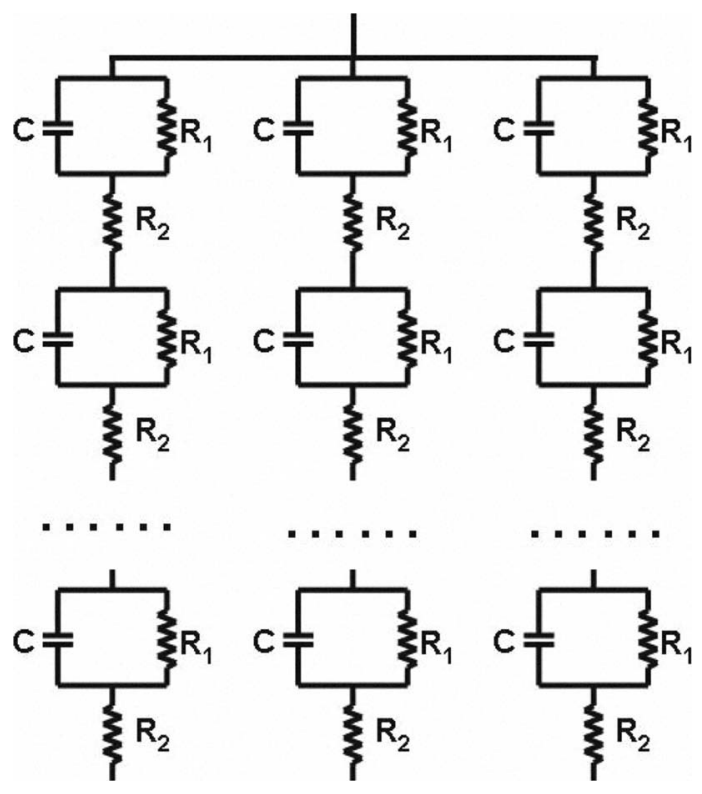

Fig. 3 Skin Impedance Model C

\section{RESULTS AND SIMULATION}

In this section, simulations based on the three skin impedance models discussed in the previous section are presented. In these simulations, a voltage is applied at time 0.1 second and it varies linearly to $5 \mathrm{~V}$ at time 0.5 second.

\section{Simulation based on Model A}

Several simulations have been carried out to examine the characteristics of the responses that may be obtained based on different parameter values. For the purpose of simulation, $R_{1}$ and $R_{2}$ have been assigned a value of $100 \mathrm{k} \Omega \mathrm{cm}^{2}$ and three different values of the capacitor have been used. Below are the results from the simulation:

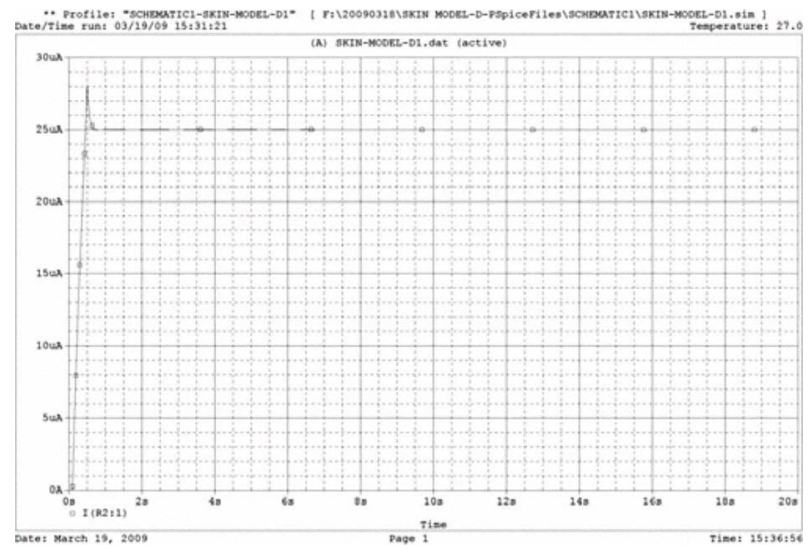

Fig. 4 Model A with capacitor value $1 \mu \mathrm{F} \mathrm{cm}{ }^{2}$ 


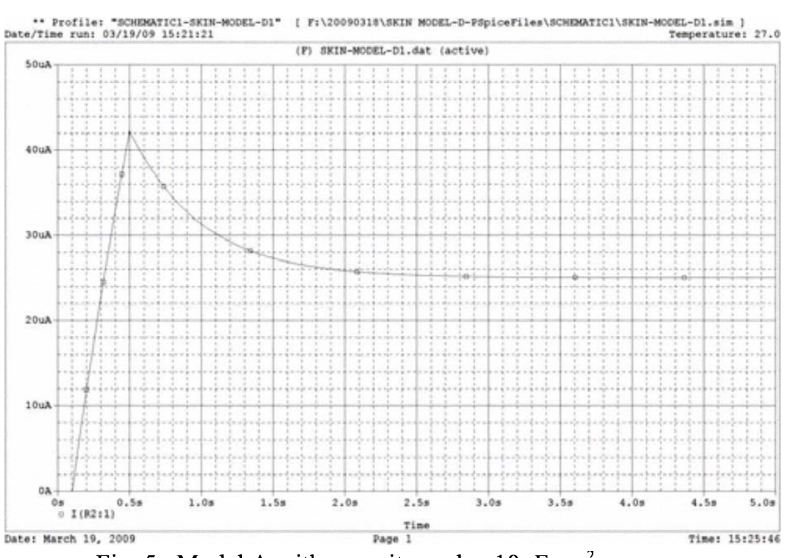

Fig. 5 Model A with capacitor value $10 \mu \mathrm{F} \mathrm{cm}^{2}$

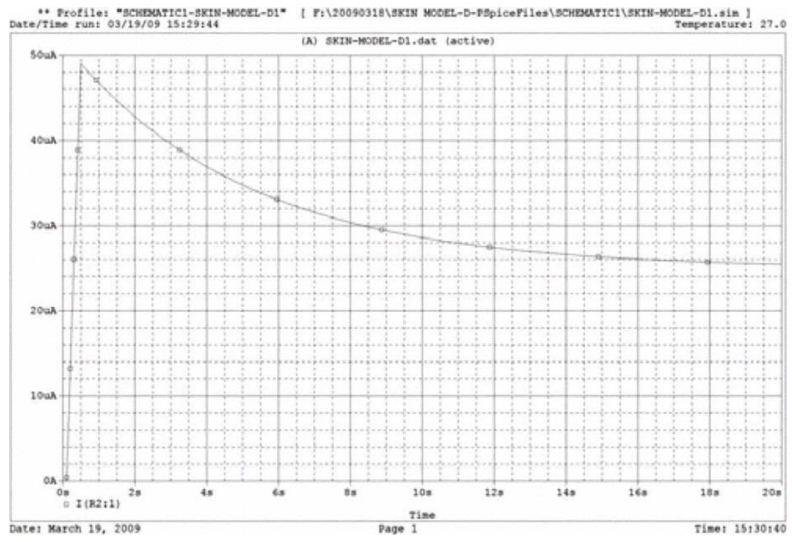

Fig. 6 Model A with capacitor value $100 \mu \mathrm{F} \mathrm{cm}{ }^{2}$

It can be seen from the simulation that as the voltage is increased from 0.1 second to 0.5 second, the current passes through the capacitor $\mathrm{C}$ of Model A. At 0.5 second, the applied voltage has already reached the final value, and the current passes through $\mathrm{R}_{1}$ and $\mathrm{R}_{2}$.

For all the three simulations, the final steady-state current depends on the value of the two resistors. As shown in Figures 4 to 6 , the value of the capacitor can have considerable effect on the shape of the response.

\section{$\underline{\text { Simulation based on Model B }}$}

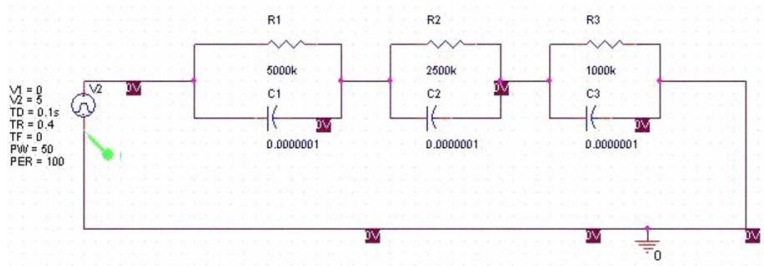

Fig. 7 Level 1 of Model B
In the first simulation of Model B, we will examine the response from Level 1. The capacitance value is $0.1 \mu \mathrm{F} \mathrm{cm}^{2}$.

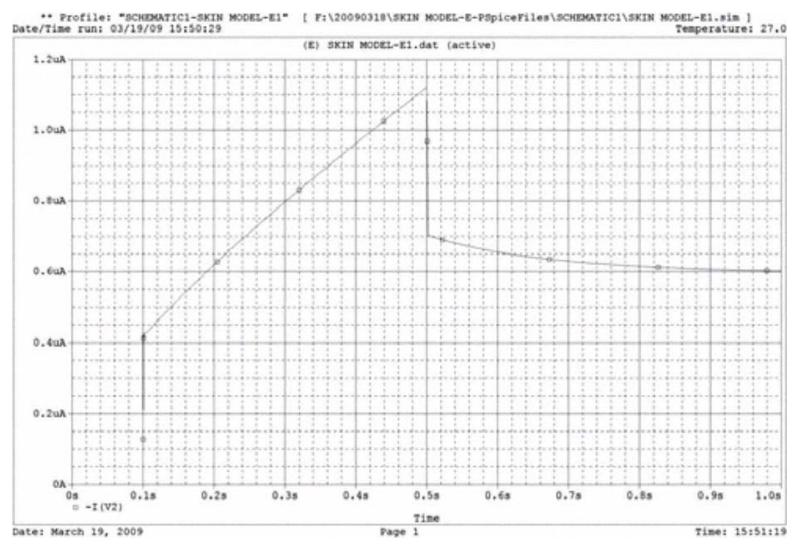

Fig. 8 Model B Level 1with capacitor value $0.1 \mu \mathrm{F} \mathrm{cm}$

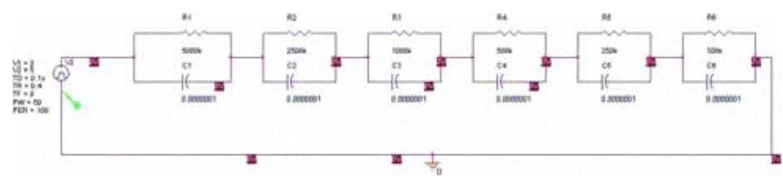

Fig. 9 Level 2 of Model B

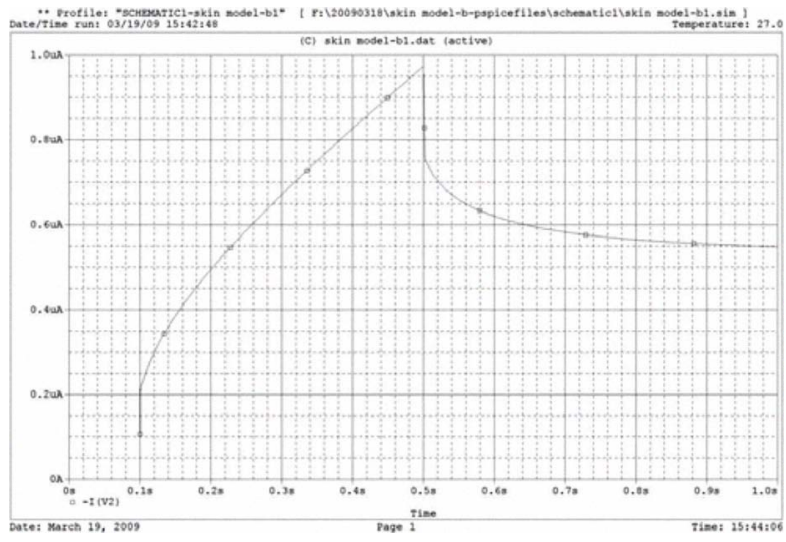

Fig. 10 Model B Level 2 with capacitor value $0.1 \mu \mathrm{F} \mathrm{cm}$

Figure 8 gives the current for Model B having only three sections (Level 1). This would model after three epidermal skin layers at different depths of the skin. Figure 9 gives the current for Model B having six sections (Level 2). More sections (or skin layers) can be included for further study, but in this paper, we show the simulations of only Level 1 and 2. As shown in Figures 8 and 10, the current can have very different response at different point of the circuit model. In both cases, the current would settle down to its steady-state level very quickly after the voltage has been steady at 0.5 second. 


\section{$\underline{\text { Simulation based on Model C }}$}

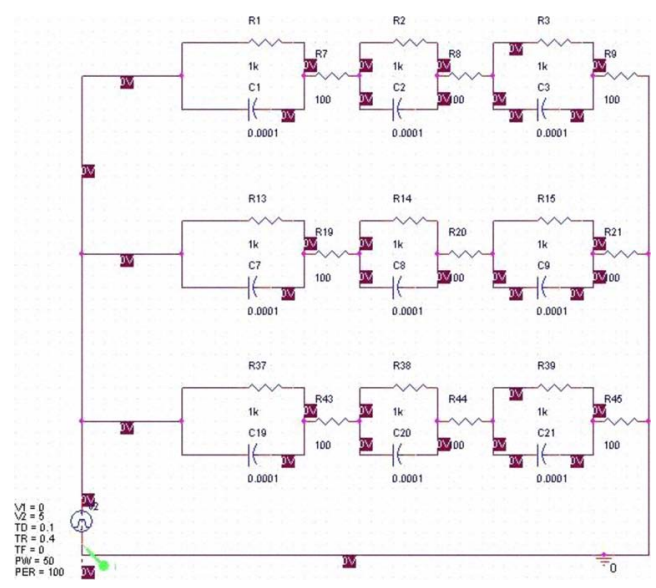

Fig. 11 Model C with three parallel paths, each with three sections

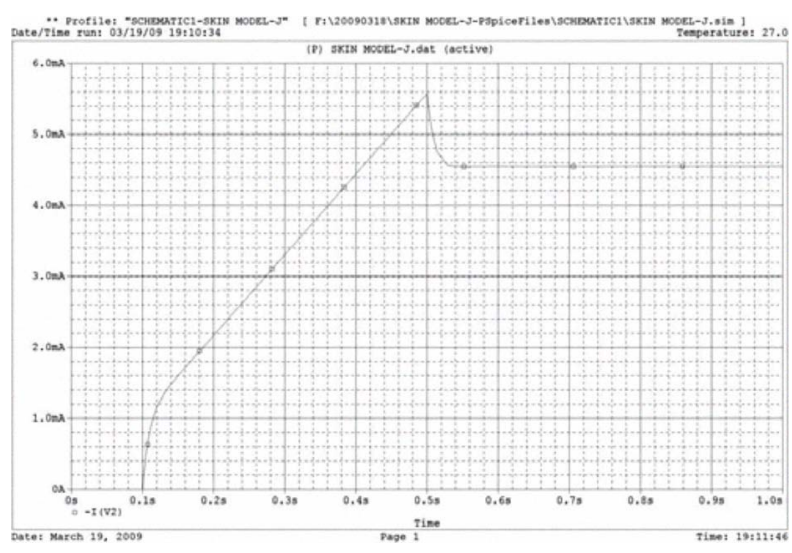

Fig. 12 Simulation result of Model C with three parallel paths, each with three sections, with capacitor value $100 \mu \mathrm{F} \mathrm{cm}{ }^{2}$ $\mathrm{R} 1=1000 \Omega \mathrm{cm} 2 \mathrm{R} 2=100 \Omega \mathrm{cm} 2$

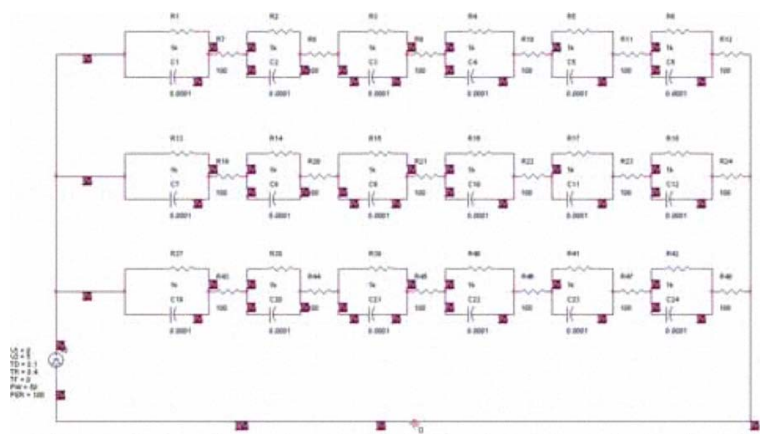

Fig. 13 Model C with three parallel paths, each with six sections

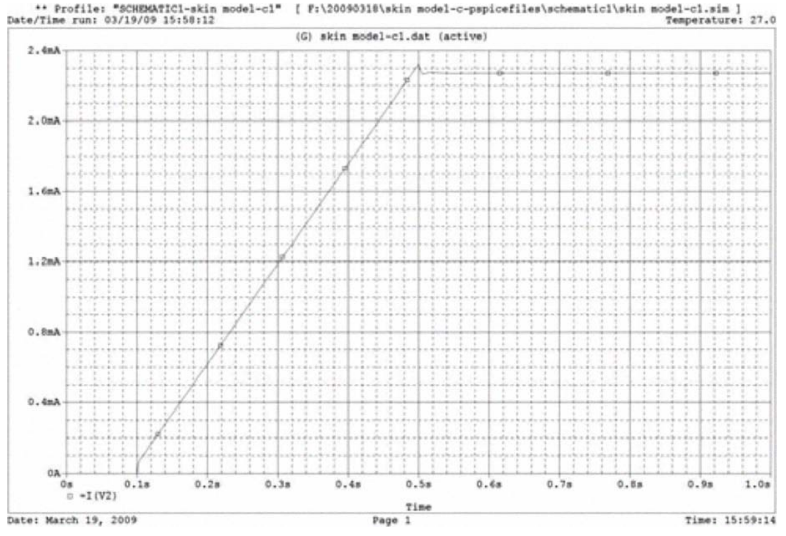

Fig. 14 Simulation result of Model C with three parallel paths, each with six sections, with capacitor value $10 \mu \mathrm{F} \mathrm{cm}$ $\mathrm{R} 1=1000 \Omega \mathrm{cm} 2 \mathrm{R} 2=100 \Omega \mathrm{cm} 2$

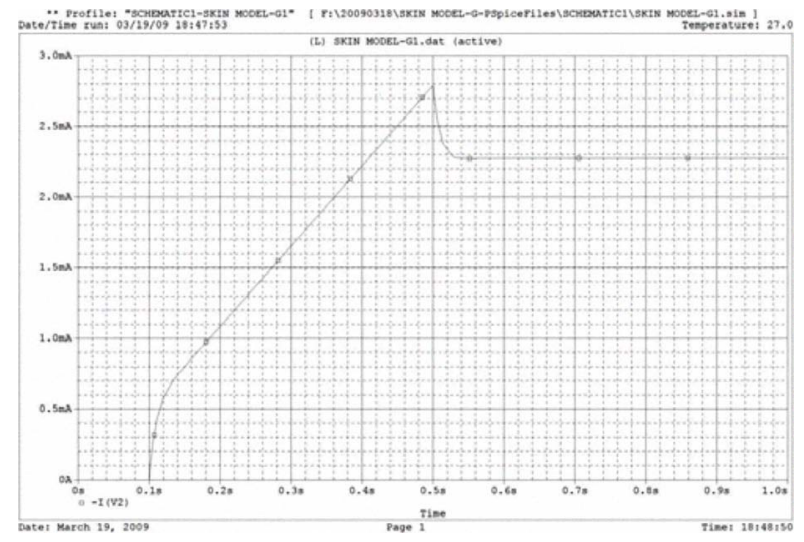

Fig. 15 Simulation result of Model C with three parallel paths, each with six sections, with capacitor value $100 \mu \mathrm{F} \mathrm{cm}{ }^{2}$ $\mathrm{R} 1=1000 \Omega \mathrm{cm} 2 \mathrm{R} 2=100 \Omega \mathrm{cm} 2$

Figure 12 and 15 are similar in shape but differ in its vertical scale. Hence, although these two results are based on different circuit models ( 3 section and 6 section model), similar response shapes are obtained with the use of different capacitor values. The vertical scales can be aligned with a careful choice of the resistor values.

\section{CONCLUSIONS}

The three-element circuit of Model A is a very simple model of the skin impedance. Considering the different characteristics to the different layer of the epidermis, the model seems not too accurate in its representation. The Model B by Tregear captures the effects of capacitance for the different layers of the epidermis. However, it is not clear how many stages should best represent the skin impedance for a certain depth of the epidermis. The model $\mathrm{C}$ by Lykken has added another resistance in between the different stage, which is used to represent deep tissue resistance. This would provide a more complete model to the skin impedance. Yet, the great variability of the parameters in the model exists and 
experimentally study is needed for a more complete understanding of the impedance characteristics of the skin.

\section{REFERENCES}

1. B. Stoebar, D. Liepmann, “Array of hollow out-of-plane microneedles for drug delivery", J. MEMS, Vol. 14, No. 3, pp. 474-479, 2005.

2. J. Ji, F.E.H. Tay, J. Miao, C. Iliescu, Microfabricated microneedles with porous tip for drug delivery, J. Micromech. Microeng., Vol. 16, pp. 958-964, 2006.

3. D.C.C. Lam, Y.H. Lee, K.T. Shek, G. Pang, "Puncture Depth and the Mechanical Stability of Microneedles", International Joint Conference on Biomedical Engineering Systems and Technologies, Biodevices 2008, Funchal, Portugal, Jan 2008.

4. A.F. Coston and J.K-J. Li, "Iontophoresis: Modeling, Methodology, and Evaluation," Cardiovasc. Engineering, Vol. 1, pp. 127-136, 2001.

5. A.F. Coston and J.K-J. Li, "Transdermal drug delivery: a comparative analysis of skin impedance models and parameters," Proc. IEEE Engineering in Medicine and Biology, Vol. 3, pp. 2982-2985, Sept 2003.

6. J.C. Lawler, M. J. Davis, E. C. Griffith, "Electrical Characteristics of the Skin: The Impedance of the Surface Sheath and Deep Tissues", J. Invest. Dermatol. 34:301-308; 1960.

7. R. Edelberg, Electrical properties of Skin In: Biophysical Properties of the Skin. Elden H. R. (ed.). New York: John Wiley \& Sons, Inc.; 1971.

8. R.T. Tregear, Physical Functions of the Skin. New York: Academic Press; 1966.

9. D.T. Lykken, "Square-Wave Analysis of Skin Impedance", Psychophysiology, 7 (2), pp. 262-275, 1970.

\section{ACKNOWLEDGEMENT}

This research is supported by a Small Project Funding grant (10400021) by the Committee on Research and Conference Grants of The University of Hong Kong. 